\title{
A Necessary and Sufficient Condition for the Non- emptiness of the Core of a Non-transferable Utility Game
}

\author{
Citation for published version (APA):
}

Predtetchinski, A., \& Herings, P. J. J. (2004). A Necessary and Sufficient Condition for the Non-emptiness of the Core of a Non-transferable Utility Game. Journal of Economic Theory, 116, 84-92. https://doi.org/10.1016/S0022-0531(03)00261-8

Document status and date:

Published: 01/01/2004

DOI:

10.1016/S0022-0531(03)00261-8

Document Version:

Publisher's PDF, also known as Version of record

\section{Please check the document version of this publication:}

- A submitted manuscript is the version of the article upon submission and before peer-review. There can be important differences between the submitted version and the official published version of record. People interested in the research are advised to contact the author for the final version of the publication, or visit the DOI to the publisher's website.

- The final author version and the galley proof are versions of the publication after peer review.

- The final published version features the final layout of the paper including the volume, issue and page numbers.

Link to publication

\footnotetext{
General rights rights.

- You may freely distribute the URL identifying the publication in the public portal. please follow below link for the End User Agreement:

www.umlib.nl/taverne-license

Take down policy

If you believe that this document breaches copyright please contact us at:

repository@maastrichtuniversity.nl

providing details and we will investigate your claim.
}

Copyright and moral rights for the publications made accessible in the public portal are retained by the authors and/or other copyright owners and it is a condition of accessing publications that users recognise and abide by the legal requirements associated with these

- Users may download and print one copy of any publication from the public portal for the purpose of private study or research.

- You may not further distribute the material or use it for any profit-making activity or commercial gain

If the publication is distributed under the terms of Article 25fa of the Dutch Copyright Act, indicated by the "Taverne" license above, 


\title{
A necessary and sufficient condition for non- emptiness of the core of a non-transferable utility game
}

\author{
Arkadi Predtetchinski and P. Jean-Jacques Herings*,1 \\ Department of Economics, Universiteit Maastricht, P.O. Box 616, 6200 MD Maastricht, The Netherlands
}

Received 26 August 2002; final version received 28 April 2003

\begin{abstract}
It is well-known that a transferable utility game has a non-empty core if and only if it is balanced. In the class of non-transferable utility games balancedness or the more general $\pi$ balancedness due to Billera (SIAM J. Appl. Math. 18 (1970) 567) is a sufficient, but not a necessary condition for the core to be non-empty. This paper gives a natural extension of the $\pi$-balancedness condition that is both necessary and sufficient for non-emptiness of the core. (C) 2003 Elsevier Inc. All rights reserved.
\end{abstract}

JEL classification: $\mathrm{C} 71$

Keywords: Cooperative games; Core; Balancedness

\section{Introduction}

A cooperative game with non-transferable utility is an assignment of a set of feasible utility allocations to each coalition of players. The core of a cooperative game selects those utility allocations that are robust to all possible deviations by coalitions.

${ }^{*}$ Corresponding author. Fax: + 31-43-3884878.

E-mail addresses: a.predtetchinski@algec.unimaas.nl (A. Predtetchinski), p.herings@algec.unimaas.nl (P. Jean-Jacques Herings).

${ }^{1}$ The research was made possible by a grant of the Netherlands Organization for Scientific Research (NWO). 
In Bondareva [2] and in Shapley [8] the notion of a balanced game has been introduced for games with transferable utility (TU-games). It has been demonstrated that a TU-game has a non-empty core if and only if it is a balanced game. Scarf [7] has extended the condition of balancedness to games with non-transferable utility (NTU-games) and proved that every balanced NTU-game has a non-empty core.

Billera [1] has further generalized the notion of a balanced game. In his work the balancedness condition is defined with respect to a system $\pi$ of coalitional vectors specifying the power or weight of each agent within every coalition. The existence of the system of the coalitional vectors $\pi$ such that the game is $\pi$-balanced suffices for the core to be non-empty. Moreover, when attention is restricted to hyperplane games, a non-empty core implies $\pi$-balancedness for an appropriate choice of $\pi$. In general, the equivalence of $\pi$-balanced games and games with a nonempty core does not hold: an example of a game with a non-empty core that is not balanced for any choice of $\pi$ is given in Billera [1]. Generalizations of Billera's balancedness conditions have been given for cooperative games in permutational structure (see [10]) and cooperative games in graph structure (see [4]). These conditions weaken $\pi$-balancedness and are sufficient for the core to be non-empty, although not necessary.

A natural generalization of the $\pi$-balancedness condition is achieved by allowing the system of coalitional vectors $\pi$ to depend on the utility allocations. In this paper we define the balancedness of the game with respect to a correspondence $\Pi$ that assigns to each allocation of utilities a set of coalitional vectors $\pi$. We therefore allow the power of an agent within a coalition to depend on the utility allocation that is proposed. We prove that the core of an NTU-game is non-empty if it is balanced with respect to some correspondence $\Pi$. Conversely, for a given game with a nonempty core we construct a correspondence $\Pi$ satisfying the condition of $\Pi$ balancedness. Thus, $\Pi$-balancedness is not only a sufficient, but also a necessary condition for non-emptiness of the core.

To the best of our knowledge, the only alternative necessary and sufficient balancedness condition for non-emptiness of the core is given by Keiding and Thorlund-Petersen [6]. The advantage of our $\Pi$-balancedness condition over the condition in Keiding and Thorlund-Petersen [6] is that it applies directly to the game of interest and avoids the construction of any auxiliary games or sequences of approximating games.

The outline of the paper is as follows. In Section 2 some notation is introduced. In Section 3 we discuss the $\pi$-balancedness condition of Billera [1] and the $(\pi,<)$-balancedness condition of Keiding and Thorlund-Petersen [6]. ${ }^{2}$ In Section 4 the $\Pi$-balancedness condition is defined and the main result of the paper is proved. In Section 5 we examine an example (due to Billera [1]) of a game which has a non-empty core without being $\pi$-balanced for any system of coalitional vectors $\pi$.

\footnotetext{
${ }^{2}$ In the paper of Keiding and Thorlund-Petersen [6] a somewhat different definition of a nontransferable utility game is given. In particular, the set $V(S)$ is assumed to lie entirely in the non-negative orthant of $\mathbb{R}^{n}$ and to contain the zero vector. The set $V_{p}(S)$ is assumed to be bounded for all $S \in \mathscr{N}$.
} 


\section{Notation}

Let $n$ be a positive integer. Then $N$ is the set of integers $\{1, \ldots, n\}$, and $\mathscr{N}$ is the collection of non-empty subsets of the set $N . \mathbb{R}^{n}$ is the space of functions $x: i \in N \mapsto x^{i} \in \mathbb{R} . \mathbb{R}^{\mathcal{N}}$ is the space of functions $\lambda: S \in \mathscr{N} \mapsto \lambda_{S} \in \mathbb{R}$. Let $\Delta_{N}$ denote the unit simplex in $\mathbb{R}^{n}, \Delta_{N}=\left\{x \in \mathbb{R}^{n} \mid x^{i} \geqslant 0 \forall i \in N, \sum_{i \in N} x^{i}=1\right\}$. For every $S \in \mathscr{N}$ define the set $\Delta_{S}=\left\{x \in \Delta_{N} \mid \sum_{i \in S} x^{i}=1\right\}$, and let $\Delta$ be a Cartesian product of $\Delta_{S}$ over all $S \in \mathscr{N}$. Let $\Delta_{\mathscr{N}}$ denote the unit simplex in $\mathbb{R}^{\mathscr{N}}, \Delta_{\mathscr{N}}=$ $\left\{\lambda \in \mathbb{R}^{\mathcal{N}} \mid \lambda_{S} \geqslant 0 \forall S \in \mathscr{N}, \sum_{S \in \mathscr{N}} \lambda_{S}=1\right\}$. If $x$ and $y$ are elements of $\mathbb{R}^{n}$, then $x \cdot y$ denotes the scalar product of $x$ and $y$, that is $x \cdot y=\sum_{i \in N} x^{i} y^{i}$. For each $S \in \mathscr{N}$ let $\mathbf{1}_{S}$ be the vector in $\mathbb{R}^{n}$ with component $\mathbf{1}_{S}^{i}$ equal to 1 if $i \in S$ and equal to 0 otherwise. For a subset $A$ of $\mathbb{R}^{n}$, the symbols int $A$ and $\partial A$ denote, respectively, the interior and the boundary of $A$.

\section{3. $\pi$-Balancedness and $(\pi,<)$-balancedness}

An n-person game with non-transferable utility (hereafter referred to simply as a game) is a family of sets $V=\langle V(S)\rangle_{S \in \mathcal{N}}$ satisfying the following assumptions:

$(\mathscr{G} 1)$ For all $S \in \mathscr{N}, V(S)$ is a non-empty proper closed subset of $\mathbb{R}^{n}$.

$(\mathscr{G})$ For all $S \in \mathscr{N},\left[x \in V(S), y \in \mathbb{R}^{n}, y^{i} \leqslant x^{i}\right.$ for all $\left.i \in S\right]$ implies $[y \in V(S)]$.

(G3) The set $V(N) \backslash \bigcup_{i \in N}$ int $V(\{i\})$ is non-empty and compact.

A vector $x \in \mathbb{R}^{n}$ is an element of the core of the game $V$ if $x \in V(N)$ and there exist no $S \in \mathscr{N}$ and no $y \in V(S)$ such that $x^{i}<y^{i}$ for all $i \in S$. The core of the game $V$ is denoted by $C(V)$. Therefore,

$$
C(V)=V(N) \backslash \bigcup_{S \in \mathcal{N}} \text { int } V(S) .
$$

Below we reproduce the $\pi$-balancedness condition of Billera [1]. Let an element $\pi$ of the set $\Delta$ be given. A collection of coalitions $\beta \subseteq \mathscr{N}$ is said to be $\pi$-balanced if there exists $\lambda \in \Delta_{\mathscr{N}}$ such that

$$
\pi_{N}=\sum_{S \in \beta} \lambda_{S} \pi_{S}
$$

A game $V$ is said to be $\pi$-balanced if for every $\pi$-balanced collection of coalitions $\beta \subseteq \mathscr{N}$ it holds that

$$
\bigcap_{S \in \beta} V(S) \subseteq V(N) \text {. }
$$

If there exists some $\pi \in \Delta$ such that the game $V$ is $\pi$-balanced, then the core of $V$ is non-empty.

The $\pi$-balancedness condition has been generalized in the work of Keiding and Thorlund-Petersen [6]. Given a game $V$ and a coalition $S \in \mathscr{N}$ let $V_{p}(S)=$ 
$\left\{x^{S} \in \mathbb{R}_{+}^{S} \mid x \in V(S)\right\}$, where $\mathbb{R}_{+}^{S}$ is the set of non-negative functions with domain $S$, and $x^{S}$ is the restriction of the function $x \in \mathbb{R}^{n}$ to the set $S .{ }^{1}$ Let $\mathscr{R}$ denote the set of binary relations $<$ on the set $\mathscr{N}$ satisfying the following conditions:

- < is acyclic: If $S_{j} \in \mathscr{N}$ for all $j \in\{1, \ldots, k\}$ and $S_{1}<S_{2}<\cdots<S_{k}$, then we cannot have $S_{k}<S_{1}$.

- All one player coalitions are minimal: For all $i \in N$ there is no $S \in \mathscr{N}$ such that $S<\{i\}$.

Let $\pi \in \Delta$ and $<\in \mathscr{R}$ be given. A game $V$ is said to be $(\pi,<)$-balanced if the following condition is satisfied: If $\beta \subseteq \mathscr{N}$ is a $\pi$-balanced collection of coalitions and

$$
x \in \bigcap_{S \in \beta} V(S) \backslash \bigcup_{Q \in \mathcal{N}, T \in \beta: Q<T} \text { int } V(Q),
$$

then $x \in V(N)$. The game $V$ is said to be weakly $(\pi,<)$-balanced if there exists a sequence $\left\{V^{\tau}\right\}_{\tau=1}^{\infty}$ of $(\pi,<)$-balanced games such that

- $V(N)=V^{\tau}(N)$ for all $\tau$, and

- for every $S \in \mathscr{N}$ the sequence $\left\{V_{p}^{\tau}(S)\right\}_{\tau=1}^{\infty}$ converges to the set $V_{p}(S)$ in the topology induced by the Hausdorff metric on the set of non-empty compact subsets of $\mathbb{R}^{n}$.

Theorem 1 below gives a characterization of NTU-games with a non-empty core.

Theorem 1 (Keiding and Thorlund-Petersen [6]). Let $V$ be a game. Then the core of $V$ is non-empty if and only if there exist $\pi \in \Delta, \quad<\in \mathscr{R}$, and a weakly $(\pi,<)$-balanced game $V^{\prime}$ such that $V(N)=V^{\prime}(N)$ and $V(S) \subseteq V^{\prime}(S)$ for all $S \in \mathscr{N}$.

The purpose of the next section is to give a necessary and sufficient condition for non-emptiness of the core that applies directly to the game of interest $V$. In particular, our condition of $\Pi$-balancedness avoids the construction of an auxiliary game like $V^{\prime}$ and does not use the approximation of the game $V^{\prime}$ by a sequence of games $\left\{V^{\tau}\right\}_{\tau=1}^{\infty}$.

\section{I-Balancedness}

As a preliminary result we state the $\Pi$-balanced version of the weak Knaster, Kuratowski, Mazurkiewicz, and Shapley $(\mathrm{K}-\mathrm{K}-\mathrm{M}-\mathrm{S})$ theorem. The original version of the $\mathrm{K}-\mathrm{K}-\mathrm{M}-\mathrm{S}$ theorem can be found in Shapley [9]. Theorem 2 can be easily established using the fixed point theorem of Kakutani (analogous to the proof of the $\mathrm{K}-\mathrm{K}-\mathrm{M}-\mathrm{S}$ theorem in Herings [3]).

Theorem 2 (П-balanced weak $\mathrm{K}-\mathrm{K}-\mathrm{M}-\mathrm{S})$. Let $\left\{C_{S} \subseteq \Delta_{N} \mid S \in \mathscr{N}\right\}$ be a closed cover of $\Delta_{N}$ such that if $S$ and $T$ are elements of $\mathscr{N}$ and the set $\Delta_{T} \cap C_{S}$ is non-empty, then $S \subseteq T$. Let $\Pi: \Delta_{N} \rightarrow \Delta$ be a convex-valued correspondence with a closed graph. Then 
there exist $x^{*} \in \Delta_{N}, \pi^{*} \in \Pi\left(x^{*}\right)$, and $\lambda^{*} \in \Delta_{\mathscr{N}}$ such that

$$
\begin{aligned}
& x^{*} \in \bigcap_{S \in \mathcal{N}: \lambda_{S}^{*}>0} C_{S}, \\
& \pi_{N}^{*}=\sum_{S \in \mathcal{N}} \lambda_{S}^{*} \pi_{S}^{*} .
\end{aligned}
$$

Definition 1. Let a game $V$ and a convex-valued correspondence $\Pi: \mathbb{R}^{n} \rightarrow \Delta$ with a closed graph be given. The game $V$ is said to be $\Pi$-balanced provided that the following condition is satisfied: If $x \in \mathbb{R}^{n}, \pi \in \Pi(x)$, and $\lambda \in \Delta_{\mathcal{N}}$ are such that

$$
\begin{aligned}
& x \in \bigcap_{S \in \mathcal{N}: \lambda_{S}>0} V(S), \\
& \pi_{N}=\sum_{S \in \mathcal{N}} \lambda_{S} \pi_{S},
\end{aligned}
$$

then $x \in V(N)$.

The $\pi$-balancedness condition of Billera [1] is defined relative to a fixed element $\pi$ of the set $\Delta$. It corresponds to the special case of Definition 1 where $\Pi$ is singlevalued and independent of the utility allocation. By using the concept of $\Pi$ balancedness, we allow the power of an agent within a coalition to depend on the utility allocation that is proposed.

Theorem 3. Let $V$ be a game. Suppose that there exists a convex-valued correspondence $\Pi: \mathbb{R}^{n} \rightarrow \Delta$ with a closed graph such that the game $V$ is $\Pi$-balanced. Then the core of the game $V$ is non-empty.

We do not report the proof of Theorem 3. Our argument essentially replicates the proof of the Scarf's theorem given in Kannai [5]. The key role in the proof of Theorem 3 is played by the $\Pi$-balanced weak $\mathrm{K}-\mathrm{K}-\mathrm{M}-\mathrm{S}$ theorem.

Theorem 4 below asserts that $\Pi$-balancedness is not only a sufficient, but also a necessary condition for the non-emptiness of the core.

Theorem 4. Let $V$ be a game with a non-empty core. Then there exists a convexvalued correspondence $\Pi: \mathbb{R}^{n} \rightarrow \Delta$ with a closed graph such that the game $V$ is $\Pi$ balanced.

Proof. We make use of the following lemma.

Lemma 1. Let $V$ be a game and let $S \in \mathscr{N}$. Then

1. for every $x \in \mathbb{R}^{n}$ there exists a unique real number $t_{S}(x)$ such that $(x-$ $\left.t_{S}(x) \mathbf{1}_{S}\right) \in \partial V(S)$

2. the function $t_{S}: x \mapsto t_{S}(x)$ is a continuous function;

3. $x \in V(S)$ if and only if $t_{S}(x) \leqslant 0 ; x \in \mathbb{R}^{n} \backslash$ int $V(S)$ if and only if $t_{S}(x) \geqslant 0$. 
Define the continuous mapping $g_{S}: \mathbb{R}^{n} \rightarrow \partial V(S)$ by $g_{S}(x)=x-t_{S}(x) \mathbf{1}_{S}$ for all $x \in \mathbb{R}^{n}$.

Let $x^{*}$ be an element of the core of the game $V$. Define the correspondence $\Pi: \mathbb{R}^{n} \rightarrow \Delta$ by letting the set $\Pi(x)$ be the product of the sets $\Pi_{S}(x)$, where

$$
\Pi_{S}(x)=\left\{\pi_{S} \in \Delta_{S} \mid \pi_{S} \cdot g_{S}\left(x^{*}\right)=\pi_{S} \cdot g_{S}(x)\right\}
$$

for all $S \in \mathscr{N}$ and $x \in \mathbb{R}^{n}$.

To see that the set $\Pi_{S}(x)$ is non-empty, observe that it is given by the intersection of the sets $\Pi_{S}^{-}(x)$ and $\Pi_{S}^{+}(x)$, where

$$
\begin{aligned}
& \Pi_{S}^{-}(x)=\left\{\pi_{S} \in \Delta_{S} \mid \pi_{S} \cdot g_{S}\left(x^{*}\right) \leqslant \pi_{S} \cdot g_{S}(x)\right\}, \\
& \Pi_{S}^{+}(x)=\left\{\pi_{S} \in \Delta_{S} \mid \pi_{S} \cdot g_{S}\left(x^{*}\right) \geqslant \pi_{S} \cdot g_{S}(x)\right\} .
\end{aligned}
$$

If the set $\Pi_{S}^{-}(x)$ were empty for some $x \in \mathbb{R}^{n}$, then the inequality $\pi_{S} \cdot g_{S}\left(x^{*}\right)>\pi_{S}$. $g_{S}(x)$ would be satisfied for all $\pi_{S} \in \Delta_{S}$. In particular, this inequality would hold for all vertices of the simplex $\Delta_{S}$, implying that $g_{S}^{i}\left(x^{*}\right)>g_{S}^{i}(x)$ for all $i \in S$. As $g_{S}\left(x^{*}\right) \in \partial V(S)$, this would imply $g_{S}(x) \in$ int $V(S)$, contradicting the definition of $g_{S}$. A similar argument shows that the set $\Pi_{S}^{+}(x)$ is non-empty. Moreover, both sets $\Pi_{S}^{-}(x)$ and $\Pi_{S}^{+}(x)$ are closed, and $\Pi_{S}^{-}(x) \cup \Pi_{S}^{+}(x)=\Delta_{S}$. Connectedness of the set $\Delta_{S}$ implies that the intersection of $\Pi_{S}^{-}(x)$ and $\Pi_{S}^{+}(x)$ is non-empty.

Convexity of the sets $\Pi_{S}(x)$ is trivial.

To prove that the graph of the correspondence $\Pi$ is closed, let $\left(x_{(k)}, \pi_{(k)}\right)$ be a sequence of points in $\mathbb{R}^{n} \times \Delta$ satisfying $\pi_{(k)} \in \Pi\left(x_{(k)}\right)$ and converging to some point $\left(x_{(0)}, \pi_{(0)}\right)$. Then the equalities

$$
\pi_{S(k)} \cdot g_{S}\left(x^{*}\right)=\pi_{S(k)} \cdot g_{S}\left(x_{(k)}\right), \quad S \in \mathscr{N}
$$

hold for all members of the sequence. By continuity of the mappings $g_{S}$, it holds in the limit that

$$
\pi_{S(0)} \cdot g_{S}\left(x^{*}\right)=\pi_{S(0)} \cdot g_{S}\left(x_{(0)}\right), \quad S \in \mathscr{N} .
$$

Therefore, $\pi_{(0)} \in \Pi\left(x_{(0)}\right)$.

Next we show that the game $V$ is $\Pi$-balanced. Let $x \in \mathbb{R}^{n}, \pi \in \Pi(x)$, and $\lambda \in \Delta_{\mathscr{N}}$ be such that

$$
\begin{aligned}
& x \in \bigcap_{S \in \mathscr{N}: \lambda_{S}>0} V(S), \\
& \pi_{N}=\sum_{S \in \mathscr{N}} \lambda_{S} \pi_{S} .
\end{aligned}
$$

By part (3) of Lemma 1, for all $S \in \mathscr{N}$ with $\lambda_{S}>0$ it holds that $t_{S}(x) \leqslant 0$. As $x^{*} \notin$ int $V(S)$, we have $0 \leqslant t_{S}\left(x^{*}\right)$. It follows that for all $S \in \mathscr{N}$ with $\lambda_{S}>0$ the following inequalities hold:

$$
\begin{aligned}
\pi_{S} \cdot x & \leqslant \pi_{S} \cdot g_{S}(x) \\
& =\pi_{S} \cdot g_{S}\left(x^{*}\right) \\
& \leqslant \pi_{S} \cdot x^{*}
\end{aligned}
$$


Therefore,

$$
\begin{aligned}
\pi_{N} \cdot x & =\sum_{S \in \mathcal{N}: \lambda_{S}>0} \lambda_{S} \pi_{S} \cdot x \\
& \leqslant \sum_{S \in \mathcal{N}: \lambda_{S}>0} \lambda_{S} \pi_{S} \cdot x^{*} \\
& =\pi_{N} \cdot x^{*} \\
& =\pi_{N} \cdot g_{N}\left(x^{*}\right) \\
& =\pi_{N} \cdot g_{N}(x)
\end{aligned}
$$

where the second to last equality is implied by the fact that $x^{*}=g_{N}\left(x^{*}\right)$. By definition of $g_{N}(x)$, this gives the inequality $t_{N}(x) \leqslant 0$, and the inclusion $x \in V(N)$.

Theorems 3 and 4 show that $\Pi$-balancedness is necessary and sufficient for nonemptiness of the core. The condition is relatively simple and provides a natural extension of $\pi$-balanced concept of Billera that closes the gap between necessary and sufficient conditions for non-emptiness of the core.

\section{An example}

In this section we examine the example from Billera [1] of a game which has a nonempty core without being $\pi$-balanced for any system of coalitional vectors $\pi$. We show that there is a natural choice of the correspondence $\Pi$ such that the game in question is $\Pi$-balanced. In addition, we demonstrate that the game is not $(\pi,<)$ balanced for any $\pi$ and any binary operation $<$, and construct an artificial game $V^{\prime}$ satisfying the condition of Theorem 1 .

Let $V$ be a game with three players where

$$
\begin{aligned}
& V(\{1,2,3\})=\left\{x \in \mathbb{R}^{3} \mid x^{1} \leqslant 1, x^{2} \leqslant 1, x^{3} \leqslant 0\right\}, \\
& V(\{1,2\})=\left\{x \in \mathbb{R}^{3} \mid x^{1}+x^{2} \leqslant 2\right\}, \\
& V(S)=\left\{x \in \mathbb{R}^{3} \mid x^{i} \leqslant 0 \text { for all } i \in S\right\} \text { for any } S \neq N,\{1,2\} .
\end{aligned}
$$

The game $V$ has a unique core-element, $(1,1,0)$.

We show that there is no $\pi \in \Delta$ such that $V$ is a $\pi$-balanced game. Suppose first that the vector $\pi_{N}$ has at least one zero component. Then the collection of singletons $\beta=\left\{\{i\} \mid \pi_{N}^{i}>0\right\}$ is a $\pi$-balanced collection of coalitions, whereas the intersection $\bigcap_{S \in \beta} V(S)$ is not a subset of $V(N)$.

Suppose that all components of the vector $\pi$ are positive. We can then assume without loss of generality that $\pi_{N}^{i}=\frac{1}{n}$ for all $i \in N$. Now, if $\pi_{\{1,2\}}^{1}=\pi_{\{1,2\}}^{2}$ then the collection of coalitions $\alpha=\{\{1,2\},\{3\}\}$ is $\pi$-balanced. Since the vector $(2,0,0)$ is an element of $V(\{1,2\})$ and $V(\{3\})$ but not of $V(\{1,2,3\})$, the condition of 
$\pi$-balancedness is violated. In case $\pi_{\{1,2\}}^{1}>\pi_{\{1,2\}}^{2}$ the collection $\beta=\{\{1,2\},\{2\},\{3\}\}$ is $\pi$-balanced, with $(2,0,0)$ being feasible for each $S$ in $\beta$. Finally, if $\pi_{\{1,2\}}^{1}<\pi_{\{1,2\}}^{2}$ then the collection of coalitions $\gamma=\{\{1,2\},\{1\},\{3\}\}$ is $\pi$-balanced. In this case the vector $(0,2,0)$ violates the $\pi$-balancedness condition.

Having these considerations in mind it is straightforward to construct a correspondence $\Pi$ such that the game $V$ is $\Pi$-balanced. In particular, there should be no $\pi$ in $\Pi(2,0,0)$ such that either $\alpha$ or $\beta$ is a $\pi$-balanced collection of coalitions. There also should be no $\pi \in \Pi(0,2,0)$ such that the collection $\gamma$ is balanced relative to $\pi$. We let

$$
\Pi_{\{1,2\}}(x)= \begin{cases}\{(a, b, 0)\} & \text { if } x^{1}<x^{2} \\ \Delta_{\{1,2\}} & \text { if } x^{1}=x^{2} \\ \{(b, a, 0)\} & \text { if } x^{1}>x^{2}\end{cases}
$$

where the numbers $a$ and $b$ satisfy $a>b \geqslant 0$ and $a+b=1$. For any coalition $S \neq\{1,2\}$ we let $\Pi_{S}(x)$ equal the barycenter of $\Delta_{S}$.

To see that $V$ is balanced relative to $\Pi$ let $x \in \mathbb{R}^{n}, \pi \in \Pi(x)$, and $\lambda \in \Delta_{\mathcal{N}}$ be such that

$$
\begin{aligned}
& x \in \bigcap_{S \in \beta} V(S), \\
& \pi_{N}=\sum_{S \in \mathcal{N}} \lambda_{S} \pi_{S},
\end{aligned}
$$

where $\beta=\left\{S \in \mathscr{N}: \lambda_{S}>0\right\}$. If $N \in \beta$ there is nothing to prove. We therefore assume that $N \notin \beta$. Suppose also that the coalition $\{1,2\}$ is not a member of the collection $\beta$. Since $\pi_{N}^{1}>0, \beta$ must contain either the coalition $\{1\}$ or the coalition $\{1,3\}$, or both. In either case $x^{1} \leqslant 0$. Similar reasoning for $x^{2}$ and $x^{3}$ shows that none of them is positive. Hence, $x \in V(N)$.

Now consider the case where the coalition $\{1,2\}$ is a member of $\beta$. If $x^{1}<x^{2}$ then, by construction of the correspondence $\Pi, \pi_{\{1,2\}}^{1}>\pi_{\{1,2\}}^{2}$. Therefore, the collection $\beta$ must contain either coalition $\{2\}$ or coalition $\{2,3\}$, or both, so $x^{2} \leqslant 0$. Since $\pi_{N}^{3}>0$, there is at least one coalition in $\beta$ which contains player 3 , so that $x^{3} \leqslant 0$. Thus, $x$ belongs to $V(N)$. If $x^{2}<x^{1}$ then $\pi_{\{1,2\}}^{2}>\pi_{\{1,2\}}^{1}$ implying that $\beta$ contains either the singleton $\{1\}$ or the coalition $\{1,3\}$. In both cases, $x^{1} \leqslant 0$. As before, we have $x^{3} \leqslant 0$. Thus, $x \in V(N)$. Finally, if $x^{1}$ equals $x^{2}$ then both of them are less than or equal to 1 , and therefore $x$ belongs to $V(N)$.

Turning to the condition of Keiding and Thorlund-Petersen, observe that the vectors $(2,0,0)$ and $(0,2,0)$ are not in the interior of any of the feasibility sets $V(S)$ of the game $V$. Hence, our argument that $V$ is not a $\pi$-balanced game for any $\pi$ is valid as a proof that it is not a $(\pi,<)$-balanced game for any $\pi$ and any $<\in \mathscr{R}$. Let $V^{\prime}$ be a game where

$$
V^{\prime}(\{i\})=\left\{x \in \mathbb{R}^{3} \mid x^{i} \leqslant 1\right\} \quad \text { for } i=1,2,
$$


and $V^{\prime}(S)=V(S)$ for all other coalitions $S$. Choose $\pi$ in $\Delta$ so that $\pi_{N}^{i}>0$ for all $i \in N$. Let $<\in \mathscr{R}$ be a binary relation satisfying $\{i\}<\{1,2\}$ for $i=1,2,3$. Then $V^{\prime}$ is a $(\pi,<)$-balanced game. Indeed, let $\beta$ be a $\pi$-balanced collection of coalitions and let $x$ be an element of the set

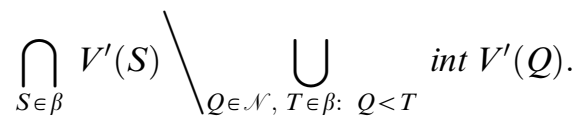

If the coalition $\{1,2\}$ is not in $\beta$ then one can argue as in the case with $\Pi$ balancedness that $x \in V^{\prime}(N)$. If $\{1,2\}$ is a member of the collection $\beta$ then $x$ must lie outside the interior of $V^{\prime}(\{i\})$ for all $i \in N$, which implies that $x^{1} \geqslant 1, x^{2} \geqslant 1$, and $x^{3} \geqslant 0$. Thus, $x=(1,1,0)$, which belongs to $V^{\prime}(N)$.

\section{Acknowledgments}

Helpful comments of Hans Peters and Andrés Perea are gratefully acknowledged.

\section{References}

[1] L.J. Billera, Some theorems on the core of an $n$-person game without side-payments, SIAM J. Appl. Math. 18 (1970) 567-579.

[2] O.N. Bondareva, Some applications of linear programming methods to the theory of cooperative games, Problemy Kibernetiki 10 (1963) 119-139 (in Russian).

[3] P.J.J. Herings, An extremely simple proof of the K-K-M-S theorem, Econ. Theory 10 (1997) 361-367.

[4] P.J.J. Herings, G. van der Laan, A.J.J. Talman, Cooperative games in graph structure, METEOR Research Memorandum 00/26, Universiteit Maastricht, 2000.

[5] Y. Kannai, The core and balancedness, in: R.J. Aumann, S. Hart (Eds.), Handbook of Game Theory with Economic Applications, North-Holland, Amsterdam, 1992, pp. 355-395.

[6] H. Keiding, L. Thorlund-Petersen, The core of a cooperative game without side payments, J. Optim. Theory Appl. 54 (1987) 273-288.

[7] H. Scarf, The core of an $n$-person game, Econometrica 35 (1967) 50-69.

[8] L.S. Shapley, On balanced sets and cores, Navel Res. Logist. Quart. 14 (1967) 453-460.

[9] L.S. Shapley, On balanced games without side payments, in: T.C. Hu, S.M. Robinson (Eds.), Mathematical Programming, Academic Press, New York, 1973, pp. 261-290.

[10] G. van der Laan, A.J.J. Talman, Z. Yang, Cooperative games in permutational structure, Econ. Theory 11 (1998) 427-442. 